\title{
Utilizing HTML-analysis and computer vision on a corpus of website screenshots to investigate design developments on the web
}

\author{
Thomas Schmidt | Anastasiia Mosiienko | Raffaela Faber | Juliane Herzog | \\ Christian Wolff
}

Media Informatics Group, University of Regensburg, Regensburg, Germany

Correspondence

Thomas Schmidt, Media Informatics Group, University of Regensburg,

Regensburg, Germany.

Email: thomas.schmidt@ur.de

\begin{abstract}
We present preliminary results of a project investigating the design development of popular websites between 1996 and 2020 via HTML analysis and basic computer vision methods. We acquired a corpus of website screenshots of the current top 47 popular websites. We crawled a snapshot of every month of these websites via the wayback machine of the Internet Archive platform since the time snapshots are stored to gather 7,953 screenshots and HTML pages. We report upon quantitative analysis results concerning HTML elements, color distributions and visual complexity throughout the years.
\end{abstract}

\section{K E Y W O R D S}

colors, html, visual complexity, web design, web history, websites

\section{1 | INTRODUCTION}

The World Wide Web has become an important part of modern media infrastructure and society. The web itself has also become an object of research in human-computer-interaction (Jørgensen \& Myers, 2008) but also cultural and media studies (Brügger, 2012). Investigating the history of web interfaces is an important task for web and media historians but can also give current web designers inspiration on how developments might continue. One important aspect of this research area is the archival of the web and the preservation of this digital heritage has been addressed by the UNESCO. ${ }^{1}$ This led to the platform Internet Archive, ${ }^{2}$ which, via its Wayback Machine ${ }^{3}$ intends to archive the web and enables design researchers to investigate trends on large-scale corpora. We present work-in-progress results of a project investigating web design developments via quantitative and qualitative analysis. We report on our current approach and the first quantitative results we acquired.

\section{2 | METHODS}

\section{1 | Corpus-acquisition}

We decided to analyze the top 50 most popular websites as of December 2019 according to the analytical platform Alexa. ${ }^{4}$ We filtered out any adult websites, which resulted in a list of 47 websites. We acquired one snapshot per day of these websites stored in the Wayback Machine, if a snapshot was available for the timespan from 1996 to 2020. A snapshot is a stored representation of the website for a given time. This results in a corpus of 151,682 snapshots. However, all websites are represented rather unequally with the most popular and oldest being most 
TA B LE 1 Websites of the corpus and number of snapshots

\begin{tabular}{|c|c|c|c|c|c|c|c|}
\hline Website & \#snapshots & Website & \#snapshots & Website & \#snapshots & Website & \#snapshot \\
\hline $\begin{array}{l}\text { Aliexpress. } \\
\text { com }\end{array}$ & 121 & Google.co.in & 194 & Okezone.com & 155 & Vk.com & 169 \\
\hline Alipay.com & 121 & Google.com.hk & 199 & qq.com & 202 & Weibo.com & 103 \\
\hline Amazon.co.jp & 161 & Google.com & 242 & Reddit.com & 179 & Wikipedia.org & 191 \\
\hline Apple.com & 242 & Jd.com & 132 & Sohu.com & 227 & $\begin{array}{l}\text { Xinhuanet. } \\
\text { com }\end{array}$ & 228 \\
\hline Babytree.com & 184 & Live.com & 102 & $\begin{array}{l}\text { Stackoverflow. } \\
\text { com }\end{array}$ & 138 & Yahoo.co.jp & 245 \\
\hline Blogspot.com & 107 & msn.com & 241 & Tmall.com & 128 & Youtube.com & 178 \\
\hline Csdn.net & 214 & Naver.com & 229 & Tribunnews.com & 110 & & \\
\hline Ebay.com & 240 & Netflix.com & 153 & Twitch.tv & 105 & & \\
\hline
\end{tabular}

TA B LE 2 Website distribution per year

\begin{tabular}{|llll|}
\hline Year & \#snapshots & Year & \#snapshots \\
\hline 1996 & 10 & 2009 & 378 \\
\hline 1997 & 23 & 2010 & 412 \\
\hline 1998 & 26 & 2011 & 487 \\
\hline 1999 & 70 & 2012 & 494 \\
\hline 2000 & 150 & 2013 & 500 \\
\hline 2001 & 183 & 2014 & 487 \\
\hline 2002 & 182 & 2015 & 491 \\
\hline 2003 & 284 & 2016 & 484 \\
\hline 2004 & 317 & 2017 & 503 \\
\hline 2005 & 349 & 2018 & 498 \\
\hline 2006 & 362 & 2019 & 501 \\
\hline 2007 & 394 & 2020 & 30 \\
\hline 2008 & 338 & & \\
\hline
\end{tabular}

frequent. For this study, we limited this corpus to one snapshot per month per website (the first available snapshot per month) to avoid problems with the unequal distribution. This subcorpus consists of 7,953 snapshots (Tables 1 and 2) of which we scraped the HTML and took a screenshot as TIFF-File with a width of 1920 pixels and height according to the size of website. To enable comparisons, we sliced the screenshots at 3000 pixels height.

The sample size for the early years is rather limited. However, beginning 2003 the sample size is more

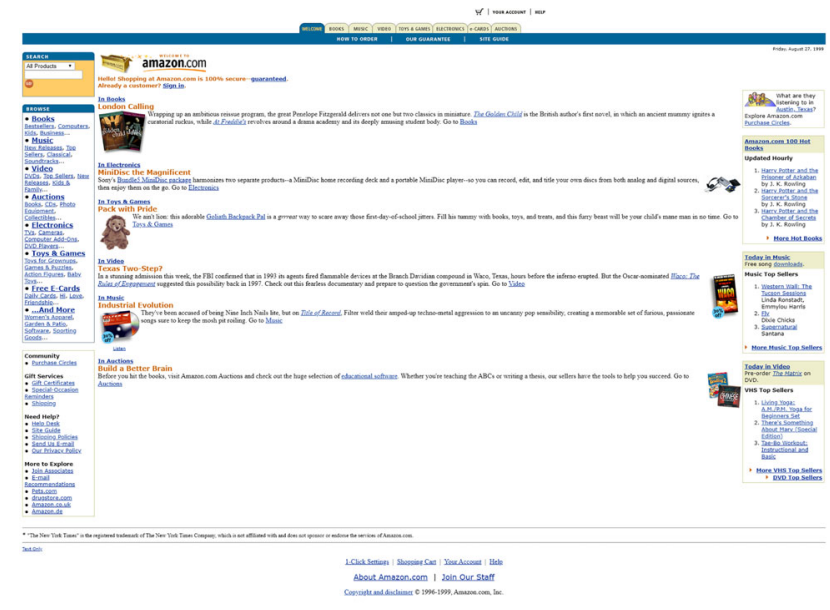

F I G U R E 1 Snapshot of Amazon.com (1998)

representative with around 300 snapshots per year. Figures 1 and 2 show two snapshots of amazon.com.

\section{2 | Analysis metrics}

We analyzed multiple quantitative metrics: (a) HTML metrics, (b) screenshot size, and (c) color metrics. We counted the number of images via the img-tag, the number of hyperlinks via the a-tag and the overall amount of words in the HTML. Another metric is the size of the screenshots measured in kilobytes after transforming 


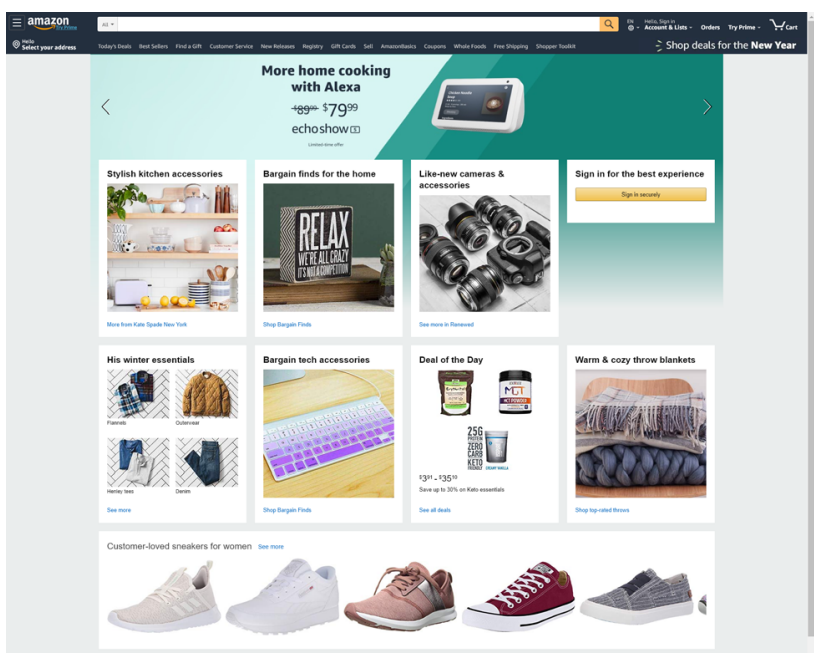

F I G U RE 2 Snapshot of Amazon.com (2020)

TABLE 3 Overview of the used RGB color ranges

\begin{tabular}{|lll|} 
Color & Lower limit $(\mathbf{R , G}, \mathbf{B})$ & Upper limit $(\mathbf{R , G}, \mathbf{B})$ \\
\hline Red & $(140,0,0)$ & $(255,56,50)$ \\
\hline Green & $(0,170,0)$ & $(130,255,70)$ \\
\hline Blue & $(0,0,145)$ & $(60,115,255)$ \\
\hline Yellow & $(230,220,0)$ & $(255,255,55)$ \\
\hline White & $(240,240,240)$ & $(255,255,255)$ \\
\hline Black & $(0,0,0)$ & $(25,25,25)$ \\
\hline
\end{tabular}

them to PNG-files. This is an established metric to measure visual complexity and has been shown to correlate with human perception of complexity (Purchase et al., 2012); the larger the more complex an image. We calculated the amount of the base colors red, green, blue, yellow as well as white and black via openCV. ${ }^{5}$ Table 3 gives an overview of the RGB-sections we included.

\section{3 | RESULTS}

We averaged the number of image-tags, hyperlink-tags and the text per year (see Figures 3-5).

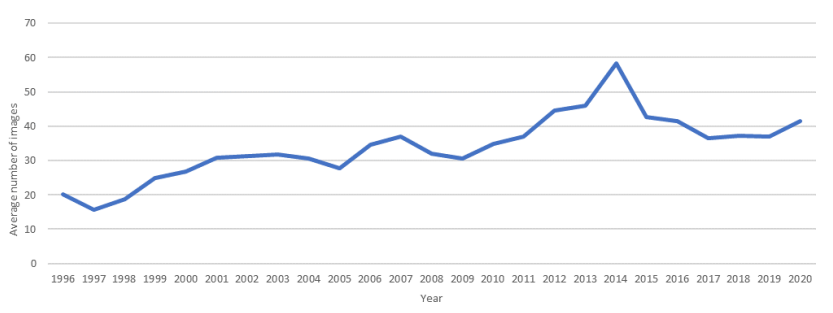

FI G URE 3 Average number of images per year

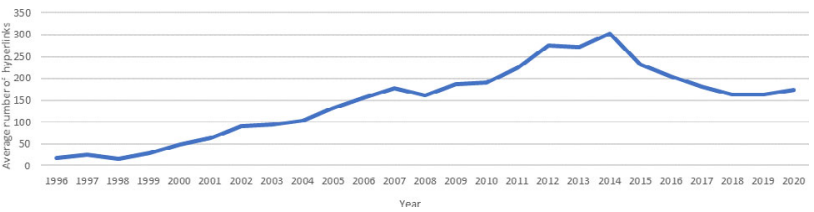

F I G U R E 4 Average number of hyperlinks per year

For the number of images and hyperlinks we can identify a steady increase up until 2014 followed by a substantial decrease. A similar development can be found for text up until 2016.

Figure 6 shows the development of the average visual complexity as measured via the filesize.

For this metric, we identified a steady increase until 2020 , with the highest leap in the time span from 2009 until 2014.

The color analysis shows that black and white are the most dominant colors since white is the general background color and black the basic font color. Figure 7 illustrates the average proportion of black and white among the websites and shows that most websites consist to around $80 \%$ percent of white. However, we observe a small decrease up until now.

While the overall proportion is much lower, we identified red and blue as the most used among the base colors, however without a consistent trend (Figure 8). Beginning with 2013, we can see a more diverse distribution among our analyzed colors. Nevertheless, without a striking trend or development. Please note that the proportions are overall very low, so the significant usage of very popular websites of one color for a year can lead to strong manifestations of this color - which is especially the case for years we do not have many snapshots for (1996-2003).

\section{4 | DISCUSSION}

We identified a steady increase of HTML-tags up until 2014 and a remarkable decrease afterwards. A similar but less strong trend is found for the number of words. We hypothesize that these results represent a trend in web design to include more content up until 2014, which is

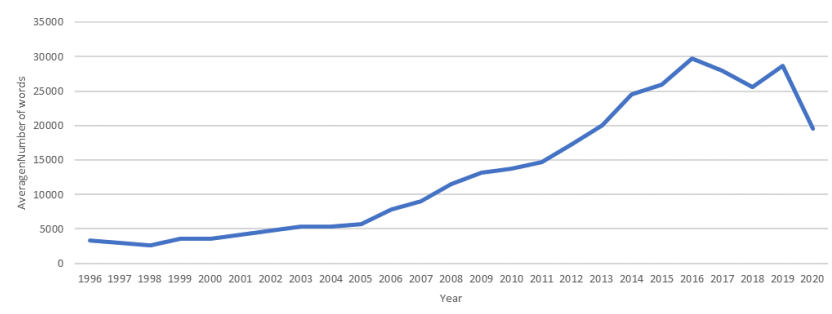

F I G URE 5 Average number of words per year 


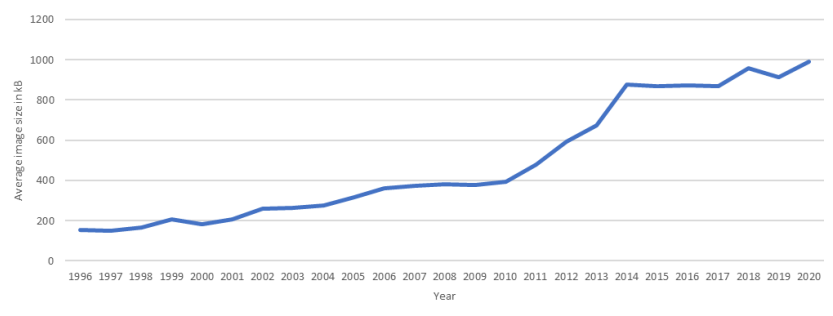

F I G U RE 6 Average size of PNG-files per year

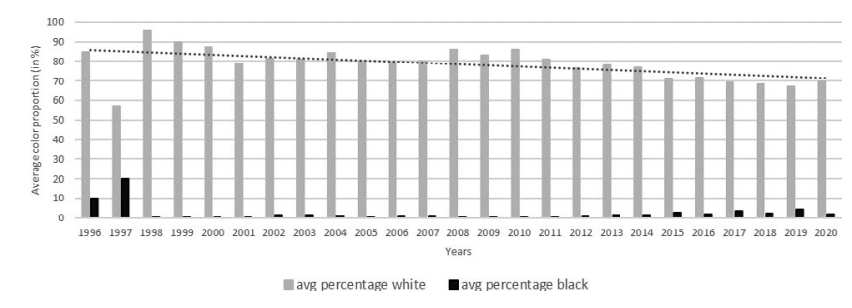

FI G URE 7 The average proportion of black and white among websites

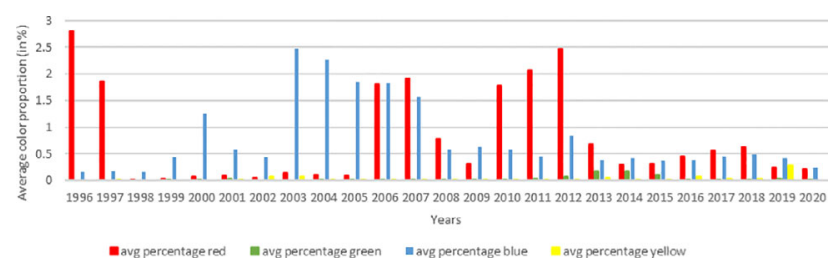

F I G URE 8 The average proportion of red, blue, green and yellow per year

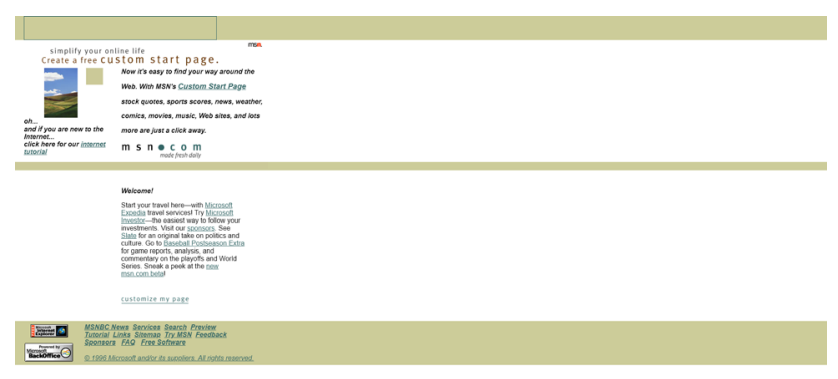

F I G U R E 9 Snapshot of Msn.com (1996)

certainly connected to increased technical possibilities. Snapshots of msn.com in our corpus are representative of this trend (Figures 9 and 10).

After 2014, this trend is followed by a tendency toward more minimalistic designs. This is opposite to the steady increase of visual complexity. The reason for the development of this metric, however, might also be the possibility for designers to include more complex images and graphs with the advent of bandwidth. While the usage of black and white is dominant on websites until now, we identified a higher usage of red and blue in early days and a more

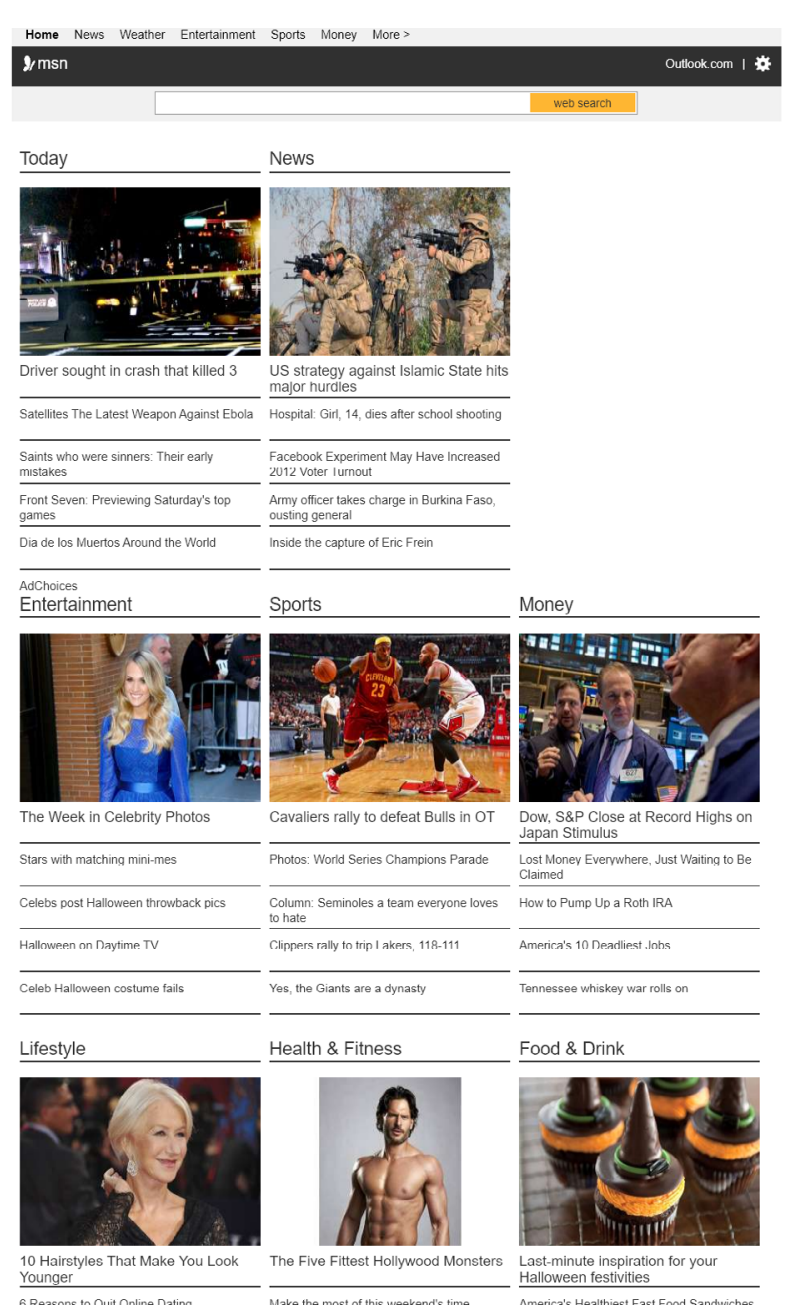

F I G U R E 10 Snapshot of Msn.com (2014)

diverse color usage beginning 2013. Our color analysis is however very limited since we neglect a wide range of other colors.

We want to continue our research by including other quantitative metrics like alternatives for visual complexity and other colors and by analyzing the websites by category. We plan to increase the corpus, especially with websites that were popular in the years 1996-2010 and are not nowadays to get a more representative sample of these times. We pursue a mixed methods approach and want to integrate qualitative analysis of a subset of the corpus to get a better understanding of design developments.

\section{ENDNOTES}

${ }^{1}$ https://en.unesco.org/themes/information-preservation/digitalheritage

2 https://archive.org/

${ }^{3}$ https://archive.org/web/

${ }^{4}$ https://www.alexa.com/

${ }^{5}$ https://opencv.org/ 


\section{REFERENCES}

Brügger, N. (2012). When the present web is later the past: Web historiography, digital history, and internet studies. Historical Social Research/Historische Sozialforschung, 37(4(142)), 102-117.

Jørgensen, A. H., \& Myers, B. A. (2008). User interface history. In CHI'08 Extended Abstracts on Human Factors in Computing Systems (pp. 2415-2418).

Purchase, H. C., Freeman, E., \& Hamer, J. (2012). An exploration of visual complexity. In International Conference on Theory and Application of Diagrams (pp. 200-213). Berlin, Heidelberg: Springer.
How to cite this article: Schmidt T, Mosiienko A, Faber R, Herzog J, Wolff C. Utilizing HTMLanalysis and computer vision on a corpus of website screenshots to investigate design developments on the web. Proc Assoc Inf Sci Technol. 2020;57:e392. https://doi.org/10.1002/ pra2.392 